\title{
Experimental study of the movement of control fluid in compound perforation
}

\author{
Zhao Xu ${ }^{1 *}$, Liu Gonghui ${ }^{1,2}$, Li Jun ${ }^{1}$ and Ding Qingxin ${ }^{3}$ \\ ${ }^{1}$ Key Laboratory of Petroleum Engineering, Ministry of Education, China University of Petroleum, Beijing 102249, China \\ ${ }^{2}$ Beijing Union University, Beijing 100010, China \\ ${ }^{3}$ School of Mechanical and Electronic Engineering, China University of Petroleum, Beijing 102249, China
}

\begin{abstract}
The interaction between the high pressure gas and the control fluid and the movement mechanism of the control fluid in compound perforation were studied by a series of large-scale experiments, where the movement behavior of the control fluid was observed. The curves of measured pressure were analyzed, a mathematical model for the rigid movement of the control fluid was established, and the movement velocity of control fluid was analyzed. Moreover, the velocity from experimental results and velocity from an analytical solution were contrasted. The movement of the control fluid in the initial stage was similar to the rigid movement; however, the propagation of the pressure wave in the control fluid should be taken into account. Experimental results are significant for research on the movement mechanism of control fluid in compound perforation.
\end{abstract}

Key words: Compound perforation, experimental study, control fluid, gas and liquid interaction, mathematical model

\section{Introduction}

Downhole compound perforation is a highly efficient completion technology, which completes perforation and high energy gas fracturing in one procedure. During the compound perforation, first of all, shaped charges are detonated to produce high speed metal particles to strike rocks, thereby making a perforation tunnel in the formation, at the same time, the propellant begins to generate gas. As the propellant burns downhole, a considerable amount of hot gas is produced that builds up a high pressure in several milliseconds (Diao et al, 2003; Sun et al, 2007). The pressure pulse is high enough to fracture downhole formations; hence it can enhance the well productivity. So far this technique has been applied to thousands of wells globally and is still being used in hundreds of wells per year. With the development of oil and gas exploration technology, low-permeability and tight sandstone reservoirs form one of the most important resources in the future petroleum production, compound perforation technology has gradually become one of the important stimulation measures to improve well productivity (Gilliat et al, 1999; Hollabaugh and Dees, 1993; Jorge et al, 2003; Li, 2002; Wang et al, 2005). This has created a great demand for research on theoretical models of compound perforation. However, relevant research is mainly concentrated on the deflagration mechanism, rock, fracturing mechanisms, and crack formation, and rarely on the movement mechanism of the control fluid (Wang and Qin, 1998; Yang and Risnes,

*Corresponding author. email: zhaoxu46@163.com

Received September 29, 2008
2000; 2001; Zhang, 2003; Lei et al, 2006; Zhang and Sun, 2006). In this paper, a new testing device was developed to investigate the movement of the top control fluid propelled by high-pressure gas. The movement of the control fluid and interaction between gas and top control fluid could be observed and measured directly, and a comparison was made between experimental results and an analytical solution from mathematical model of rigid movement, providing an experimental basis for oil field implementation.

\section{Experimental}

\subsection{Basic thinking}

An experimental device was designed based on the geometric similarity and dynamical similarity principles. To ensure safe operation in our experiments, high-pressure gas was used instead of explosive charges. Pressure transducers were used to measure the variation in pressure and an $\mathrm{A} / \mathrm{D}$ card was used to gather and record pressure data.

\subsection{Experimental materials and method}

In practical compound perforation, the pressure of the high energy gas ranges from 40 to $70 \mathrm{MPa}$, even reaching $100 \mathrm{MPa}$, due to different fracturing charge properties and perforation conditions. At last, a large amount of high-energy gas goes through the control fluid and escapes from the well, and the other is forced into the formation. The experimental device designed to investigate the movement of the top control fluid propelled by high-pressure gas is illustrated in Fig. 1. 


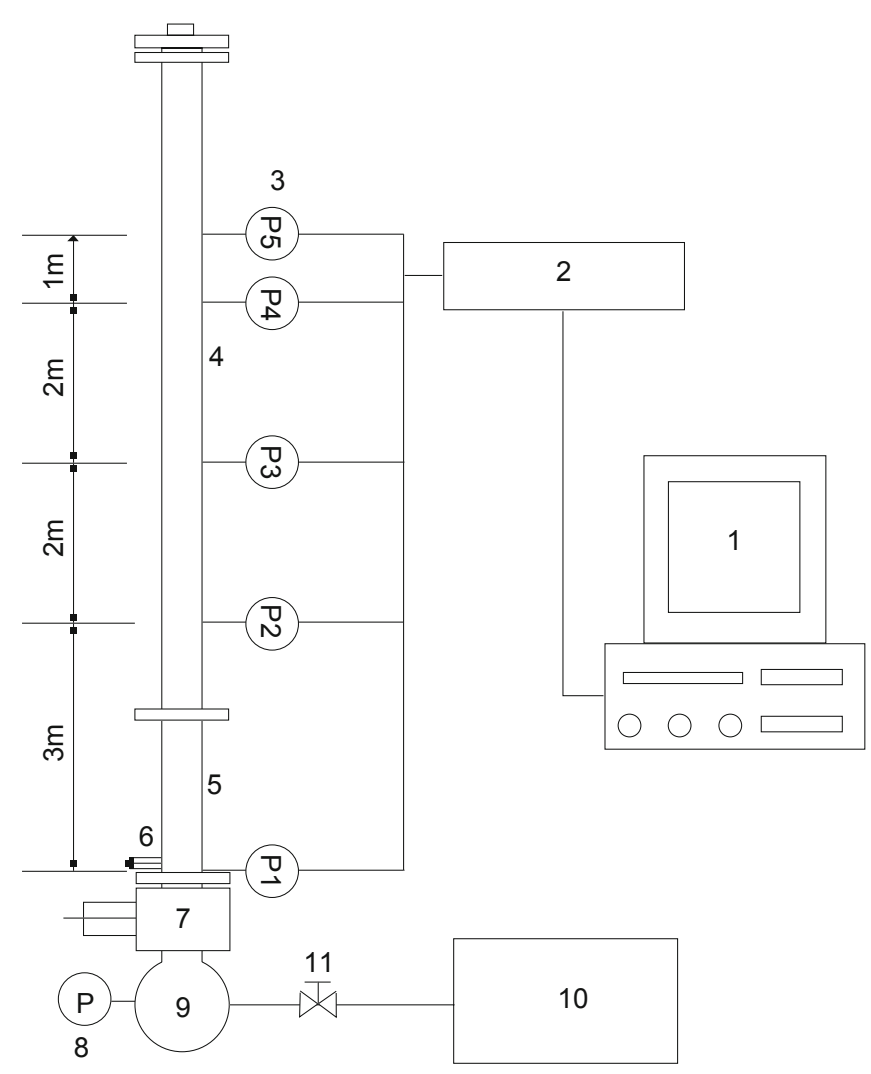

Fig. 1 Experimental system

1-Computer; 2-NI6009 card; 3-Pressure transducer; 4-Packer; 5-Plexiglas pipe; 6-Steel pipe; 7-Security valve; 8-Quick-opening valve; 9-Pressure gauge; 10 -Air chamber; 11-Depressuring valve; 12-Gas-holder

The testing device mainly included four parts: highpressure gas generation system, simulated pipeline, a variable volume gas holder, and a data acquisition and processing system. The simulated pipeline, with an inner diameter of 30 $\mathrm{mm}$, was $11 \mathrm{~m}$ long and comprised of 9-meter Plexiglas pipe and 2-meter steel pipe. The steel pipe was installed under the Plexiglas pipe. The gas-liquid interaction could be observed through the Plexiglas pipe and the security of the highpressure gas could be guaranteed by the initial section of steel pipe. A high-pressure gas generation system was installed in the bottom of the simulated pipe. It connected with a quickopening valve that could be opened completely in several milliseconds, so the high-pressure gas could act on top control fluid instantaneously. The maximum volume of the gas-holder was $157 \mathrm{~mL}$ and the minimum value $30 \mathrm{~mL}$ (Zhao et al, 2009).

Five pressure transducers were sensitive and solid enough to measure static and dynamic pressure. They had a bandwidth of approximately $1,000 \mathrm{~Hz}$. The transducer for measuring borehole pressure was mounted at the cell seat with its sensor head pointing directly into the borehole. An NI6009 PC-based logging system was chosen for the measuring system. It was an integrated data processor with eight parallel channels of sampling. The system had a maximum sampling frequency of $48 \mathrm{kHz}$ and could be used for a wide range of measurement operations. A simple real-time software package was installed in a PC for controlling the logging system.

\subsection{Downhole pressure simulation and experimental parameters}

It is important to ensure that the experimental conditions are similar to those occurring downhole during compound perforation. The most influential factors are high-energy gas pressure, control fluid height, and gas volume. Considering the applicability of the experimental device and the experimental aim, two groups of experiments were conducted on similar principles, and experimental parameters are listed in Table 1.

Table 1 Relevant experimental parameters

\begin{tabular}{|c|c|c|c|c|c|c|}
\hline \multirow{2}{*}{ Test No. } & \multicolumn{3}{|c|}{ First group } & \multicolumn{3}{|c|}{ Second group } \\
\hline & $\begin{array}{c}\text { Gas pressure } \\
\mathrm{MPa}\end{array}$ & $\begin{array}{l}\text { Control fluid height } \\
\text { m }\end{array}$ & $\begin{array}{c}\text { Tolerance } \\
\mathrm{mL}\end{array}$ & $\begin{array}{c}\text { Gas pressure } \\
\mathrm{MPa}\end{array}$ & $\begin{array}{l}\text { Control fluid height } \\
\mathrm{m}\end{array}$ & $\begin{array}{c}\text { Tolerance } \\
\mathrm{mL}\end{array}$ \\
\hline 1 & 3 & 10 & 157 & 4 & 10 & 140 \\
\hline 2 & 3 & 10 & 140 & 4 & 10 & 130 \\
\hline 3 & 3 & 10 & 130 & 4 & 10 & 120 \\
\hline 4 & 3 & 10 & 120 & 4 & 10 & 110 \\
\hline 5 & 3 & 10 & 110 & 4 & 10 & 100 \\
\hline 6 & 3 & 10 & 100 & 4 & 10 & 90 \\
\hline 7 & 3 & 10 & 90 & 4 & 10 & 80 \\
\hline 8 & 3 & 10 & 80 & 4 & 10 & 70 \\
\hline 9 & 3 & 10 & 70 & 4 & 10 & 60 \\
\hline 10 & 3 & 10 & 60 & 4 & 10 & 50 \\
\hline 11 & 3 & 10 & 50 & 4 & 10 & 40 \\
\hline 12 & 3 & 10 & 40 & 4 & 10 & 30 \\
\hline
\end{tabular}




\section{Experimental results and discussion}

\subsection{The characteristics of gas pressure in the initial stage}

A packer of variable diameter was installed at the outlet of the simulated pipe, therefore, when the top control fluid rushed out of the simulated pipe, a reverse shock wave would be formed. Hence, through collecting the pressure data of the shock wave, the time required for control fluid to pass through the simulated pipe and to rush out of the pipe could be obtained. And the average velocity of the control fluid in the whole interaction process was also obtained. For analyzing the characteristics of high-energy gas pressure both in the bottom of the pipe and control fluid, two groups of experiments were conducted. In the first group of experiments, the gas volumes were 40 and $157 \mathrm{~mL}$, respectively, and the gas pressure was kept constant at $3 \mathrm{MPa}$; and in the second group of experiments, the gas volumes were 30 and $140 \mathrm{~mL}$, respectively, and the gas pressure was kept constant at $4 \mathrm{MPa}$.

Fig. 2 shows pressures measured at different positions

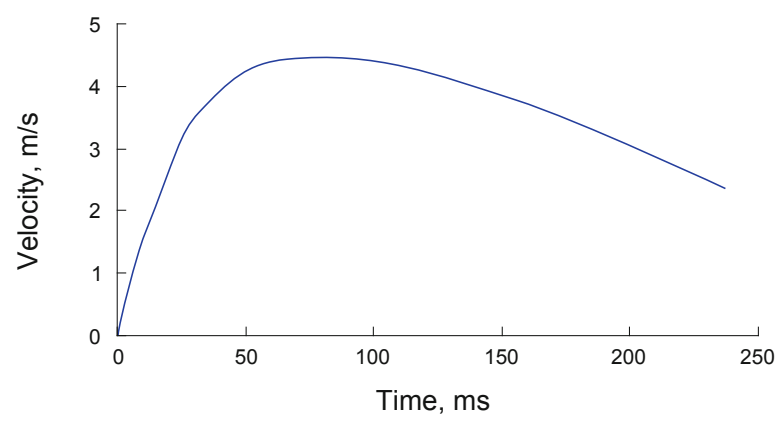

(a) High pressure gas: $3 \mathrm{MPa}$; tolerance: $157 \mathrm{~mL}$

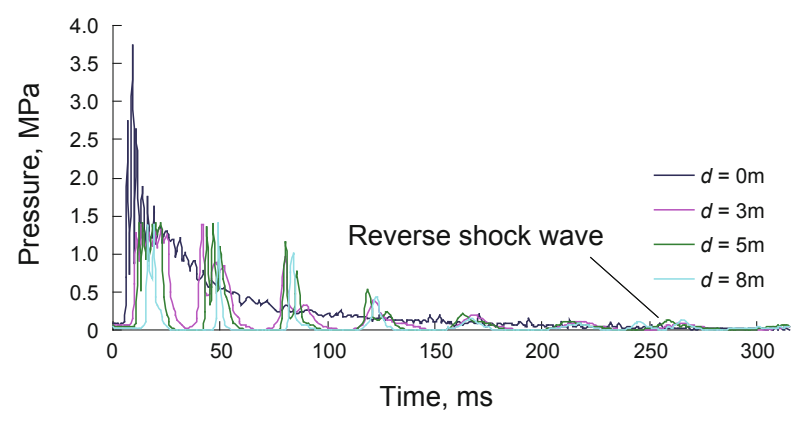

(c) High pressure gas: $3 \mathrm{MPa}$; tolerance: $40 \mathrm{~mL}$ of the simulated pipe. In Fig. 2, $d$ refers to the distance from the pressure-measuring point to the bottom of the pipe. It can be found from Fig. 2 that the pressure in the simulated pipe increased rapidly because of the instantaneous shock of the bottom high-pressure gas, followed by a rapid drop. The process of sharp fluctuation in pressure lasted almost $50 \mathrm{~ms}$. After $50 \mathrm{~ms}$, the pressure gradually became steady, however, the fast and small-scale pressure fluctuation always existed. A sudden pressure change was observed when the fluid column moved to the outlet of the simulated pipe. High bottom gas pressure and large gas volume would result in an obvious reverse pressure wave and a long duration of high bottom gas pressure. Otherwise, when the bottom gas pressure was low and the gas volume was small, the reverse pressure wave was very small and inconspicuous and the duration time of high bottom gas pressure was short. The pressures in the control fluid measured by different pressure transducers was correlated. The logging system acquired the data from five transducers simultaneously, there was a time delay, on the order of several milliseconds, between pressure waves received by adjacent transducers located at different distances from the bottom of the simulated pipe.

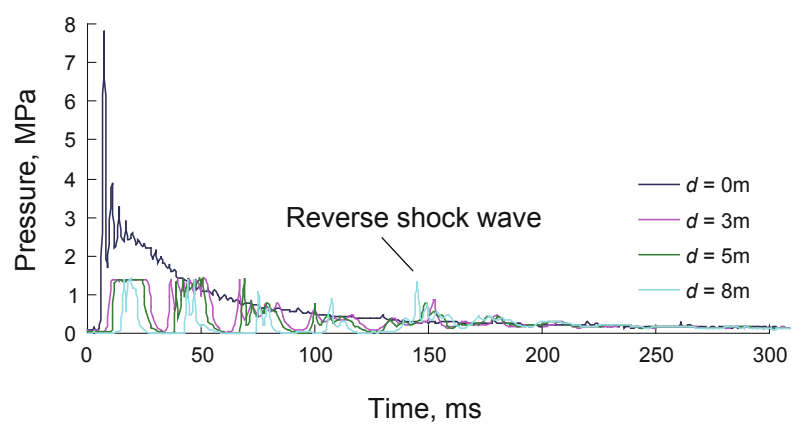

(b) High pressure gas: $4 \mathrm{MPa}$; tolerance: $140 \mathrm{~mL}$

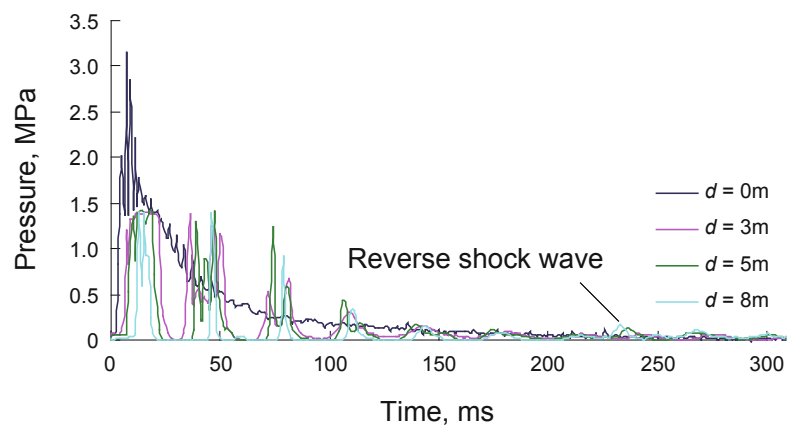

(d) High pressure gas: $4 \mathrm{MPa}$; tolerance: $30 \mathrm{~mL}$

Fig. 2 Characteristics of pressure variation in the initial stage

\subsection{Computational model for rigid movement of control fluid}

Through analyzing the above-mentioned experimental results, we could draw a conclusion that the movement of the control fluid was similar to rigid movement. A computational model for the rigid movement of the control fluid was proposed.
As shown in Fig. 3, the rigid displacement of the fluid column in the wellbore can be determined by using Newton's second law, therefore, the shape function can be expressed as follows (Xiang, 2002; Li et al, 1972):

$$
\frac{\mathrm{d}(m v)}{\mathrm{d} t}=\left(P(t)-P_{\mathrm{a}}\right) S-m g-F
$$


where $m=\rho H S$ is the mass of the top control fluid; $S$ is the cross-sectional area of the simulated pipe; $H$ is the height of fluid column; $\rho$ is the density of the control fluid; $g$ is the acceleration of gravity; $v$ is the velocity of the fluid column; $P(t)$ is the pressure measured by the bottom pressure transducer; $P_{\mathrm{a}}$ is the local atmospheric pressure; $F$ is the viscous resistance caused by friction between the fluid column and the pipe wall, and it can be expressed as follows (Yuan, 2004):

$$
F=\lambda \frac{H}{D} \frac{\rho v^{2}}{2} \frac{\pi D^{2}}{4}=\frac{\lambda \pi D H \rho v^{2}}{8}
$$

Substituting Eq. (2) into Eq. (1) gives:

$$
\frac{\mathrm{d} v}{\mathrm{~d} t}=\frac{P(t)-P_{\mathrm{a}}}{\rho H}-g-\frac{\lambda v^{2}}{2 D}
$$

where $\lambda$ is the friction coefficient; $D$ is the inner diameter of the simulated pipe.

In experiments, the height of the fluid column $H$ was 10 $\mathrm{m}$; and the inner diameter of the simulated pipe $D$ was $0.03 \mathrm{~m}$. Since the inner surface of the simulated pipe (steel pipe and Plexiglas pipe) had been treated specially, its surface finish was very high and its roughness was very low. Therefore,

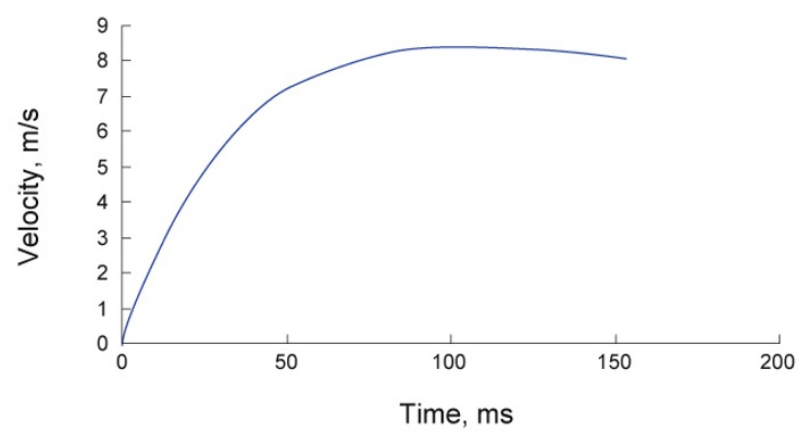

(a) High pressure gas: $3 \mathrm{MPa}$; tolerance: $157 \mathrm{~mL}$

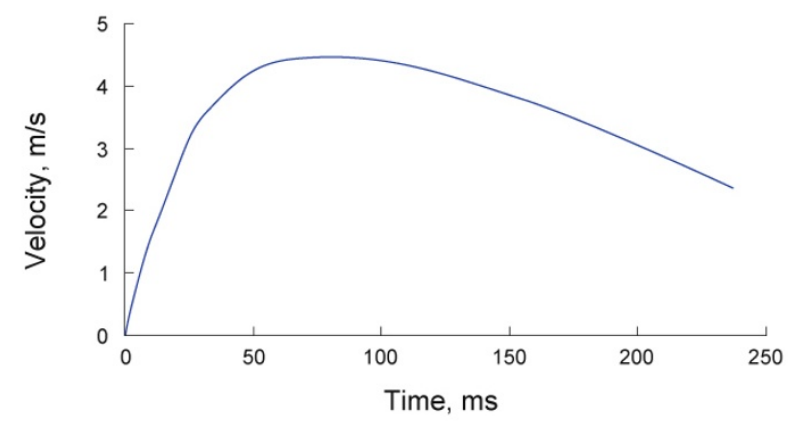

(c) High pressure gas: $3 \mathrm{MPa}$; tolerance: $40 \mathrm{~mL}$ the simulated pipe was a hydraulically smooth pipe. For a hydraulically smooth pipe, the friction coefficient $\lambda$ is a function of the Reynolds number. The velocity of the top control fluid measured in these experiments was $0-10 \mathrm{~m} / \mathrm{s}$, for the sake of simplicity, the value of $\lambda$ was set at 0.018 (Wu, 2003). Therefore, the relationship between movement velocity and time can be achieved, as shown in Fig. 4.

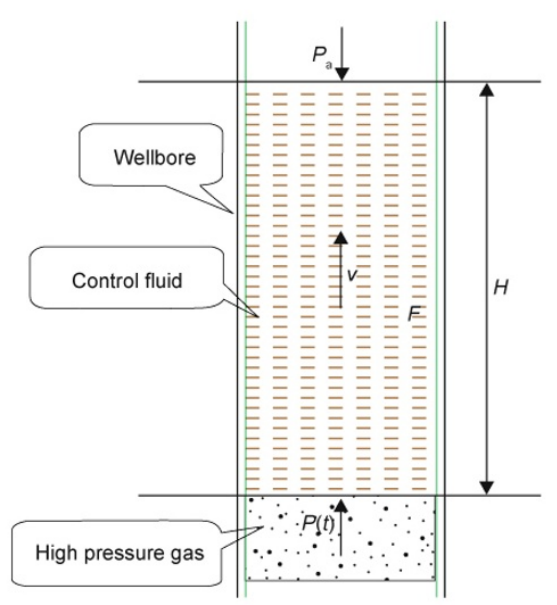

Fig. 3 Schematic diagram of the movement of the control fluid

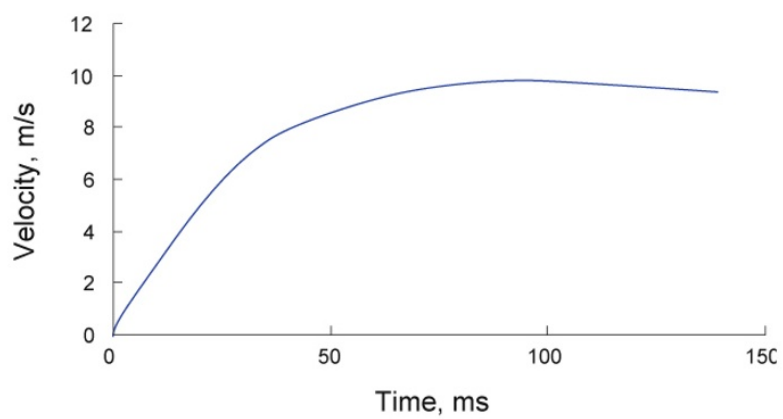

(b) High pressure gas: $4 \mathrm{MPa}$; tolerance: $140 \mathrm{~mL}$

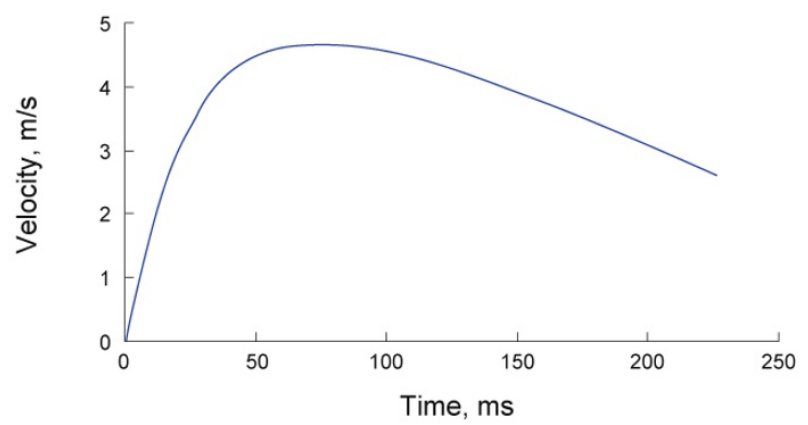

(d) High pressure gas: $4 \mathrm{MPa}$; tolerance: $30 \mathrm{~mL}$

Fig. 4 Characteristics of movement velocity of the control fluid in the initial stage

Fig. 4 shows velocity-time curves of the control fluid in the simulated pipe, which were calculated by Eq. (3). The calculated time is the duration required for the control fluid to move to the outlet of simulated pipe. It can be seen from Fig. 4 that the control fluid moves up rapidly under the action of the bottom high pressure gas, and its movement velocity reaches a peak value after about $50 \mathrm{~ms}$ and subsequently changes slightly. Finally the movement velocity of the control fluid begins to decline due to the limited volume of highpressure gas. Fig. 4 also shows that the gas volume and 
gas pressure have distinct influences on the control fluid movement. Large gas volume and high bottom gas pressure will significantly increase movement velocity and prolong the duration of the steady stage, as shown in Fig. 4(a) and Fig. 4(b). Therefore, the time required for the control fluid to move to the outlet of the simulated pipe is short, as shown in Fig. 4. The movement time is $153 \mathrm{~ms}$ under the conditions illustrated on Fig. 4(a), however, its value is $236 \mathrm{~ms}$ under the conditions illustrated on Fig. 4(c). This indicates that when using a large volume of gas or bottom gas of high pressure, the energy of the bottom gas is comparatively large, it makes the velocity of the control fluid increase very quickly. With the control fluid moving upwards, the volume occupied by the bottom gas increases, then the pressure of the bottom gas declines, but the bottom gas still can propel the control fluid upwards for a long time at a steady high-speed. Otherwise, when using a small volume of gas and bottom gas of relatively low pressure, the whole energy of bottom gas is too small. Therefore, after acting on the control fluid, the bottom gas loses energy quickly, and the bottom gas pressure declines rapidly. This makes the velocity of control fluid increase for only a very short time, followed by a gradual drop.

\subsection{Comparison between the analytical solution and experimental results}

In experiments, the height of control fluid was $10 \mathrm{~m}$, and the lengths of simulated pipe and packer of variable diameter were 11 and $0.098 \mathrm{~m}$, respectively. Therefore, the distance that the control fluid moved upwards was $1.098 \mathrm{~m}$. The duration of time that the control fluid passed this distance could be obtained according to the pressure data measured by transducers installed at different positions along the pipe. The average velocity of control fluid can be calculated by the following equation:

$$
\overline{V_{1}}=\frac{L}{t}
$$

where $\bar{V}_{1}$ is average velocity, $\mathrm{m} / \mathrm{s} ; L$ is the movement distance, $L=1.098 \mathrm{~m} ; t$ is the time required for control fluid to pass through the pipe of $1.098 \mathrm{~m}$.

Hence, the average velocities calculated from experimental data are listed in Table 2.

By using Eq. (3), the change of movement velocity of the fluid column with time can be calculated, furthermore, the average velocity of the control fluid in the whole interaction time can be calculated by the following equation:

$$
\overline{V_{2}}=\int_{0}^{t} v \mathrm{~d} t
$$

where $\overline{V_{2}}$ is the average velocity derived from the analytical solution, $\mathrm{m} / \mathrm{s} ; v$ is the velocity from Eq. (3), $\mathrm{m} / \mathrm{s}$.

Therefore, the average velocities from experimental results and from analytical solution were obtained from Eq. (4) and Eq. (5) respectively and a comparison between them are shown in Fig. 5.

\begin{tabular}{|c|c|c|c|c|}
\hline \multirow[b]{2}{*}{ Test No } & \multicolumn{2}{|c|}{ First group } & \multicolumn{2}{|c|}{ Second group } \\
\hline & $\begin{array}{l}\text { Time } \\
\text { ms }\end{array}$ & $\begin{array}{c}\text { Average velocity } \\
\mathrm{m} / \mathrm{s}\end{array}$ & $\begin{array}{c}\text { Time } \\
\text { ms }\end{array}$ & $\begin{array}{c}\text { Average velocity } \\
\mathrm{m} / \mathrm{s}\end{array}$ \\
\hline 1 & 153 & 7.72 & 138 & 7.96 \\
\hline 2 & 158 & 6.95 & 140 & 7.84 \\
\hline 3 & 163 & 6.74 & 142 & 7.73 \\
\hline 4 & 167 & 6.57 & 150 & 7.32 \\
\hline 5 & 171 & 6.42 & 151 & 7.27 \\
\hline 6 & 176 & 6.24 & 157 & 6.99 \\
\hline 7 & 189 & 5.81 & 163 & 6.74 \\
\hline 8 & 192 & 5.72 & 165 & 6.65 \\
\hline 9 & 202 & 5.44 & 181 & 6.07 \\
\hline 10 & 214 & 5.13 & 189 & 5.81 \\
\hline 11 & 225 & 4.88 & 204 & 5.38 \\
\hline 12 & 236 & 4.65 & 226 & 4.86 \\
\hline
\end{tabular}

Table 2 Experimental results

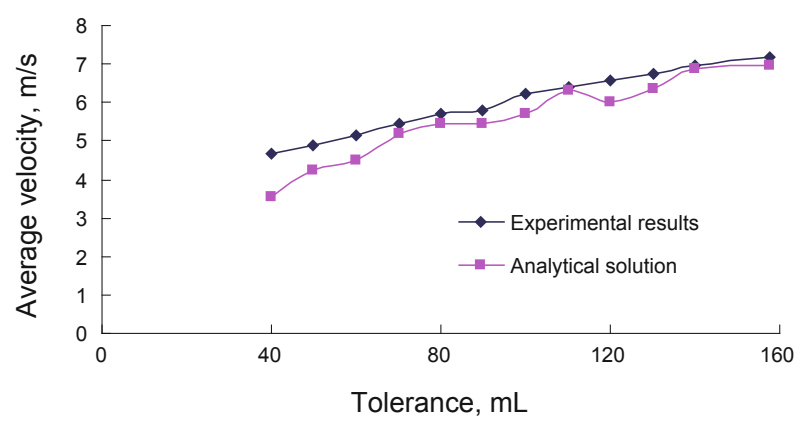

(a) High gas pressure: $3 \mathrm{MPa}$ (The first group experiments)

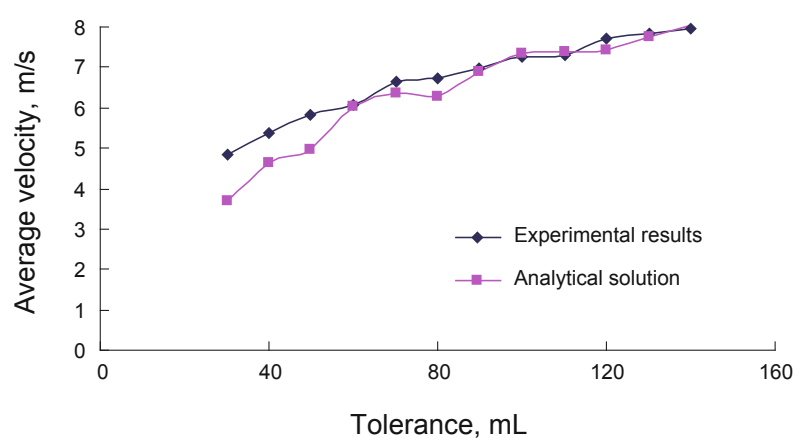

(b) High pressure gas: 4MPa (The second group experiments)

Fig. 5 Velocity contrast between the analytical solution and experimental results

Fig. 5 shows that the calculated values were consistent with measured values. When the volume of high pressure gas was large, the error between the calculated and measured 
values was small, but with a decrease in gas volume, the error consistently increased. This indicated that with a decrease in bottom gas, the time that the control fluid was lifted by high propellant gas pressure decreased, and the energy of highpressure gas decayed very quickly. Therefore, the control fluid could not be lifted up to the outlet of pipe in the effective interaction time. In the last stage, the gas pressure that was applied to the top control fluid was too small, the control fluid had already started to move under inertia. At this time, the bottom gas pressure has not been the force that propelled the control fluid to move up. If the pressure data that were measured from the bottom pressure transducer were used to calculate the velocity of the control fluid, a big error would be induced. Fig. 2(c) and Fig. 2(d) also illustrated that the reverse shock wave was comparatively small when the gas volume was not too large. Both the pressure on the control fluid and the velocity of the control fluid decreased when the control fluid moved up to the outlet of the pipe. Fig. 4(a) and Fig. 4(b) showed that the error between the analytical solution and experimental results was comparatively small when the gas volume was large, nearly all the values from the analytical solution were smaller than experimental results. That is because the location at which the bottom pressure transducer installed was some distance from the high-pressure gas generation device, the pressure values measured at this location were a little lower than the force applied to the top control fluid. Therefore, the average velocity from analytical solution was lower than the experimental results.

From the analysis above, it can be concluded that the movement of the top control fluid during compound perforation is similar to rigid movement in the initial stage. The mathematical model for rigid movement (Eq. (3)) can describe the movement of the control fluid during compound perforation. Because of the limitation of experimental conditions, the height of control fluid was only $10 \mathrm{~m}$ and the pressure of gas was 3-4 MPa. However, the propagation of the pressure wave was still measured, the pressure wave in the fluid column spread in the form of successive layers. In an actual wellbore, the fluid column is usually several thousands meters high, and a period of time is required for the pressure wave to propagate through the whole fluid column. Therefore, in the compound perforation design, the propagation of the pressure wave in the fluid column should taken into account, after the pressure wave propagates through the whole fluid column, the movement of control fluid can be described as a rigid movement.

\section{Conclusions}

1) The interaction between the high pressure gas and the control fluid is a complicated process during compound perforation; both the pressure in the control fluid and the bottom gas pressure fluctuate rapidly in the initial stage.

2) The movement of control fluid is similar to rigid movement during compound perforation, but the propagation of pressure wave in the fluid column has an important influence.

3) The movement of the control fluid was analyzed with a mathematical model. The movement of the control fluid can be divided three stages: acceleration, steady, and deceleration. The movement velocity and the duration of the steady velocity increase with the increases in the gas pressure and gas volume.

\section{References}

Diao G T, Liu Z H and Zhou J J. The application of compound perforation technology. Drilling \& Production Technology. 2003. 26(6): 39-42 (in Chinese)

Gilliat J, Snider P M and Haney R L. A review of field performance of new propellant/perforating technologies. SPE Annual Technical Conference and Exhibition held in Houston, Texas, 3-6 October 1999. (SPE paper 56469)

Hollabaugh G S and Dees J M. Propellant gas fracture stimulation of a horizontal Austin chalk wellbore. 68th Annual Technical Conference and Exhibition of the Society of Petroleum Engineers held in Houston, Texas, 3-6 October 1993 (SPE paper 26584)

Jorge B, Edgar A, Kent F, el al. Propellant perforation breakdown technique: Eastern Venezuela field application. SPE International Improved Oil Recovery Conference in Asia Pacific held in Kuala Lumpur, Malaysia, 20-21 October 2003 (SPE paper 84913)

Lei W, Zhang G and Du L. Study of the application of composite perforation technology in the west Sichuan Basin. Natural Gas Industry. 2006. 26(5): 58-60 (in Chinese)

Li K M. High power, multiplex perforation techniques and their prospects. Petroleum Drilling Techniques. 2002. 30(3): 72-74 (in Chinese)

Li W X, Han Z H and Zheng K Q. Differential Equations and Application of Hydromechanics. Shanghai: Shanghai Science and Technology Press. 1972. 162-170 (in Chinese)

Sun X B, Liu H and Wang B X. Review of propellant perforation techniques. Explosive Materials. 2007. 36(5): 29-31 (in Chinese)

Wang A S and Qin F D. High Energy Gas Fracturing Technology. Xi'an, Shaanxi: Northwest University Press. 1998. 66-145 (in Chinese)

Wang H D, Sun X B, Liang C. A summary of perforating technology development inside and outside of China. Explosive Materials. 2005. 35(3): 33-36 (in Chinese)

Wu C G. Hydromechanics. Beijing: Higher Education Press. 2003. 3872 (in Chinese)

Xiang Z H. Study on Perforation Fracturing Parameter Optimization. MSc Thesis. Beijing: China University of Petroleum. 2002 (in Chinese)

Yang D W and Risnes R. Experimental study on fracture initiation by pressure pulses. SPE Annual Technical Conference and Exhibition held in Dallas, Texas, 1-4 October 2000 (SPE paper 63035)

Yang D W and Risnes R. Numerical modelling and parametric analysis for designing propellant gas fracturing. SPE Annual Technical Conference and Exhibition held in New Orleans, Louisiana, 30 September-3 October 2001 (SPE paper 71641)

Yuan E X. Engineering Fluid Mechanics. Beijing: Oil Industry Press. 2004. 1-10 (in Chinese)

Zhang G A and Sun Z M. Application of fracGun in an overseas field. Petroleum Drilling Techniques. 2006. 31(01): 62-64 (in Chinese)

Zhang X S. Study of applications of composite perforation technology and high energy gas fracturing in oilfield. Heihe Science and Technology. 2003. (2): 20-22 (in Chinese)

Zhao X, Liu G H, Zhang T. Movement mechanism of control fluid in propellant perforation under the action of down high-pressure gas. Acta Petrolei Sinica. 2009. 30 (2): 295-299 (in Chinese) 